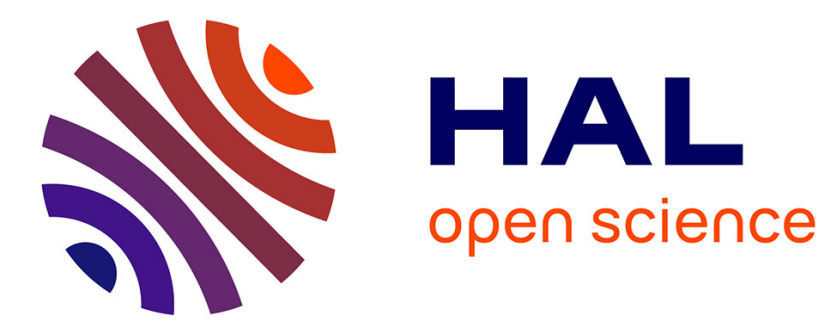

\title{
BURNING NUMBER OF GRAPH PRODUCTS
}

Dieter Mitsche, Pawel Pralat, Elham Roshanbin

\section{To cite this version:}

Dieter Mitsche, Pawel Pralat, Elham Roshanbin. BURNING NUMBER OF GRAPH PRODUCTS.

Theoretical Computer Science, 2017. hal-02083877

\section{HAL Id: hal-02083877 \\ https://hal.science/hal-02083877}

Submitted on 29 Mar 2019

HAL is a multi-disciplinary open access archive for the deposit and dissemination of scientific research documents, whether they are published or not. The documents may come from teaching and research institutions in France or abroad, or from public or private research centers.
L'archive ouverte pluridisciplinaire HAL, est destinée au dépôt et à la diffusion de documents scientifiques de niveau recherche, publiés ou non, émanant des établissements d'enseignement et de recherche français ou étrangers, des laboratoires publics ou privés. 


\title{
BURNING NUMBER OF GRAPH PRODUCTS
}

\author{
DIETER MITSCHE, PAWE€ PRAŁAT, AND ELHAM ROSHANBIN
}

\begin{abstract}
Graph burning is a deterministic discrete time graph process that can be interpreted as a model for the spread of influence in social networks. The burning number of a graph is the minimum number of steps in a graph burning process for that graph. In this paper, we consider the burning number of graph products. We find some general bounds on the burning number of the Cartesian product and the strong product of graphs. In particular, we determine the asymptotic value of the burning number of hypercube graphs and we present a conjecture for its exact value. We also find the asymptotic value of the burning number of the strong grids, and using that we obtain a lower bound on the burning number of the strong product of graphs in terms of their diameters. Finally, we consider the burning number of the lexicographic product of graphs and we find a characterization for that.
\end{abstract}

\section{INTRODUCTION}

Graph burning is a graph process that models the spread of influence in social networks and was introduced in $[3,4,8]$. Here is the definition of this process which is defined on the node set of a simple finite graph. There are discrete time-steps (or rounds) and initially all nodes are unburned. In the first round, we choose one node that catches fire. At the beginning of every round $t(t \geq 2)$, the fire spreads from the set of burning nodes to their unburned neighbours. Then we choose one node and start the fire there, unless the node is already on fire. (Of course, choosing a node that is already on fire is usually suboptimal but we allow this to avoid complications with situations in which no unburned node is available.) Throughout the process, each node is either burned or unburned. Once a node is burned it remains in that state until the end of the process. The process ends at the end of round $T$ when all nodes are burning.

Suppose that we burn a graph $G$ in $k$ steps in a burning process. For $1 \leq i \leq k$, the node $x_{i}$ that we choose to burn directly in the $i$-th step of this process is called the $i$-th fire source. The sequence $\left(x_{1}, \ldots, x_{k}\right)$ is called a burning sequence for $G$. The burning number of a graph $G$, written by $b(G)$, is the length of a shortest burning sequence for $G$. Such a burning sequence is called an optimum burning sequence for $G$. For example, it is easy to see that $b\left(C_{4}\right)=2$; the sequence $\left(v_{1}, v_{3}\right)$ is an optimum burning sequence for $C_{4}$, as shown in Figure 1. The red nodes and edges demonstrates the fire spread from $v_{1}$ and the blue node is the fire started

The second author was partially supported by NSERC. 
at $v_{3}$. The burning number can be used as a measure for the speed of spreading fire on the node set of graphs.

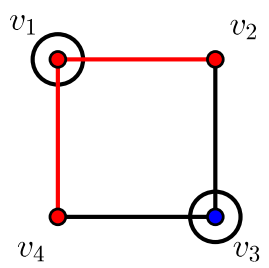

Figure 1. An optimum burning sequence for $C_{4}$.

Given two graphs $G$ and $H$, one can create a new graph on the node set $V(G) \times V(H)$. There are several different ways to define the connections (or the edges) of such a graph, and they have been studied well in the theory of graphs; see [6]. Since the burning number is a relatively new parameter, it is natural to consider the burning number of graph products. Several facts and bounds on the burning number of graphs are given in $[1,4,8]$. It is shown in $[2,8]$ that the graph burning problem is NP-complete even for trees and path-forests. Some probabilistic results on the burning number of graphs, and some random variations of graph burning are presented in $[7,8]$. In this paper, we consider the burning number of graph products and its relation to the burning number of the initial graphs.

\section{PRELIMINARIES}

We first present some terminology, and then we review some known facts about graph burning and the burning number that are needed throughout the paper. For a node $v$ in a graph $G$, the eccentricity of $v$ is defined as $\max \{d(v, u): u \in V(G)\}$. The radius of $G$, denoted by $\operatorname{rad}(G)$, is the minimum eccentricity of a node in $G$. The center of $G$ is the set of the nodes in $G$ with minimum eccentricity. The diameter of $G$, denoted by $\operatorname{diam}(G)$, is the maximum eccentricity over the node set of $G$. For a positive integer $k$, the $k$-th closed neighbourhood of node $v$, denoted by $N_{k}[v]$, is defined to be the set $\{u \in V(G): d(u, v) \leq k\}$. We sometimes use the notation $N_{k}^{G}[v]$ to emphasize that we consider the $k$-th closed neighbourhood of node $v$ in a specified graph $G$.

The Cartesian product of two graphs $G$ and $H$, denoted by $G \square H$, is the graph with node set $V(G) \times V(H)$, in which two nodes $\left(u_{1}, v_{1}\right)$ and $\left(u_{2}, v_{2}\right)$ are adjacent if and only if, either $u_{1}=u_{2}$ and $v_{1} v_{2} \in E(H)$, or $u_{1} u_{2} \in E(G)$ and $v_{1}=v_{2}$. The strong product of two graphs $G$ and $H$, denoted by $G \otimes H$, is the graph with node set $V(G) \times V(H)$, in which two nodes $\left(u_{1}, v_{1}\right)$ and $\left(u_{2}, v_{2}\right)$ are adjacent if and only if $v_{1} v_{2} \in E(H)$ or $u_{1} u_{2} \in E(G)$. It is known that $d_{G \otimes H}\left(\left(u_{1}, v_{1}\right),\left(u_{2}, v_{2}\right)\right)=\max \left\{d_{G}\left(u_{1}, u_{2}\right), d_{H}\left(v_{1}, v_{2}\right)\right\}$ (see, for example, [6]). By definition, we get immediately that $G \square H \subseteq G \otimes H$. 
The lexicographic product of two graphs $G$ and $H$, denoted by $G \circ H$, is the graph with node set $V(G) \times V(H)$, in which two nodes $\left(u_{1}, v_{1}\right)$ and $\left(u_{2}, v_{2}\right)$ are adjacent if and only if either $u_{1} u_{2} \in E(G)$, or $u_{1}=u_{2}$ and $v_{1} v_{2} \in E(H)$. In other words, $G \circ H$ is isomorphic to the graph that is constructed by replacing each node $u_{i}$ in $G$ by a copy of $H$, called $H_{i}$, and then adding all edges $u v$, where $u \in V\left(H_{i}\right), v \in V\left(H_{j}\right)$, and $u_{i} u_{j}$ is an edge in $G$. Namely, for $1 \leq i \leq|V(G)|$, $V\left(H_{i}\right)=\left\{\left(u_{i}, v\right): v \in V(H)\right\}$. If $d_{G}\left(u_{i}, u_{l}\right)$ and $d_{H}\left(v_{j}, v_{s}\right)$ are finite (that is, $u_{i}, u_{j}$ belong to the same connected component of $G$ and $v_{j}, v_{s}$ are in the same component of $H)$, then for the nodes $\left(u_{i}, v_{j}\right)$ and $\left(u_{l}, v_{s}\right)$ in $G \circ H$, the following holds: if $u_{i} \neq u_{l}$, then

$$
d_{G \circ H}\left(\left(u_{i}, v_{j}\right),\left(u_{l}, v_{s}\right)\right)=d_{G}\left(u_{i}, u_{l}\right) ;
$$

if $u_{i}=u_{l}$ and $v_{j} \neq v_{s}$, then

$$
d_{G \circ H}\left(\left(u_{i}, v_{j}\right),\left(u_{l}, v_{s}\right)\right)=\min \left\{2, d_{H}\left(v_{j}, v_{s}\right)\right\} .
$$

For more on graph products see, for example, [6].

A subgraph $H$ of a graph $G$ is called an isometric subgraph if for every pair of nodes $u$ and $v$ in $H$, we have that $d_{H}(u, v)=d_{G}(u, v)$. For example, a subtree of a tree is an isometric subgraph. Also, if $G$ is a connected graph and $P$ is a shortest path connecting two nodes of $G$, then $P$ is an isometric subgraph of $G$.

Here are some facts about the burning number from $[3,8]$ that we need for proving the results in this paper. From the definition of the burning process we can easily conclude the following lemma which is equivalent to Lemma 1 in [4].

Lemma 1. A sequence $\left(x_{1}, x_{2}, \ldots, x_{k}\right)$ forms a burning sequence for a graph $G$ if and only if

$$
N_{k-1}\left[x_{1}\right] \cup N_{k-2}\left[x_{2}\right] \cup \ldots \cup N_{0}\left[x_{k}\right]=V(G)
$$

and

$$
N_{k-2}\left[x_{1}\right] \cup N_{k-3}\left[x_{2}\right] \cup \ldots \cup N_{0}\left[x_{k-1}\right] \neq V(G) .
$$

The above lemma shows that a graph burning process for $G$ is in fact a problem of covering the node set of $G$.

Theorem 2 ([8]). If for some $t \leq k, C_{1}, C_{2}, \ldots, C_{t}$ is a collection of connected subgraphs in a graph $G$ with radii $k-1, k-2, \ldots, k-t$, respectively, which cover all nodes of $G$, then $b(G) \leq k$.

Indeed, (1) is satisfied with $x_{i}$ being a center of subgraph $C_{i}$ (that is, any vertex $x_{i}$ such that all vertices of $C_{i}$ are at distance at most $k-i$ from $x_{i}$.)

Note that if $H$ is a spanning subgraph of a graph $G$, then $b(G) \leq b(H)$ (see $[4,8])$.

Theorem 3 ([4]). Suppose that $H$ is an isometric subgraph of a graph $G$ such that, for any node $x \in V(G) \backslash V(H)$, and any positive integer $r$, there exists a node $f_{r}(x) \in V(H)$ for which $N_{r}[x] \cap V(H) \subseteq N_{r}^{H}\left[f_{r}(x)\right]$. Then we have that $b(H) \leq b(G)$. 
Theorem 4 ([4]). For any isometric subtree $H$ of a graph $G$, we have that $b(H) \leq$ $b(G)$.

Theorem 5 ([4]). A graph $G$ of order $n$ satisfies $b(G)=2$ if and only if $G$ is of order at least 2 , and has maximum degree $n-1$ or $n-2$.

Theorem 6 ([4]). For a path $P_{n}$ on $n$ nodes, we have that $b\left(P_{n}\right)=\left\lceil n^{1 / 2}\right\rceil$.

\section{MAin Results}

In this section, we present our main results as follows. We first state two simple general bounds on the burning number of the Cartesian product and the strong product of graphs. We then find the asymptotic value of the burning number of hypercube graphs, and we state a conjecture on the exact value. We also consider the asymptotic value of the burning number of strong grids; using that we obtain a lower bound on the burning number of the strong product of graphs in terms of their diameters. We finish this paper by characterizing the burning number of the lexicographic product of graphs.

We start with bounds on the burning number of the Cartesian product and the strong product of two graphs.

Theorem 7. If $G$ and $H$ are two connected graphs, then we have that

$\max \{b(G), b(H)\} \leq b(G \otimes H) \leq b(G \square H) \leq \min \{b(G)+\operatorname{rad}(H), b(H)+\operatorname{rad}(G)\}$.

Proof. First, note that $G \square H$ is a spanning subgraph of $G \otimes H$. Thus,

$$
b(G \otimes H) \leq b(G \square H) .
$$

For proving the lower bound of $b(G \otimes H)$, note that each of $G$ and $H$ is an isometric subgraph of $G \otimes H$ that satisfies the conditions in Theorem 3. To see this, suppose that $V(G)=\left\{u_{1}, \ldots, u_{m}\right\}$ and $V(H)=\left\{v_{1}, \ldots, v_{n}\right\}$, for some positive integers $m$ and $n$. Let $u_{k}$, where $1 \leq k \leq m$, be a central node in $G$. By definition of the strong product, the subgraph of $G \otimes H$ induced by the set of the nodes $\left\{\left(u_{k}, v_{i}\right): 1 \leq i \leq n\right\}$ is isomorphic to $H$. We denote this subgraph by $H_{k}$. We claim that, for any node $\left(u_{i}, v_{j}\right)$ in $G \otimes H$ and a positive integer $r$, we have that

$$
N_{r}^{G \otimes H}\left[\left(u_{i}, v_{j}\right)\right] \cap H_{k} \subseteq N_{r}^{H_{k}}\left[\left(u_{k}, v_{j}\right)\right] .
$$

To see this, let $\left(u_{k}, v_{l}\right) \in V\left(H_{k}\right)$, where $1 \leq l \leq n$, with $d_{G \otimes H}\left(\left(u_{k}, v_{l}\right),\left(u_{i}, v_{j}\right)\right) \leq r$. Then we have that

$$
\begin{aligned}
r \geq d_{G \boxplus H}\left(\left(u_{k}, v_{l}\right),\left(u_{i}, v_{j}\right)\right) & =\max \left\{d_{G}\left(u_{k}, u_{i}\right), d_{H}\left(v_{l}, v_{j}\right)\right\} \\
& \geq d_{H}\left(v_{l}, v_{j}\right) \\
& =d_{G \otimes H}\left(\left(u_{k}, v_{l}\right),\left(u_{k}, v_{j}\right)\right) .
\end{aligned}
$$

Hence, $H$ is an isometric subgraph of $G \otimes H$ that satisfies the conditions in Theorem 3. Similarly, by symmetry of the strong product, we can conclude that 
$G$ is also an isometric subgraph of $G \otimes H$ that satisfies the conditions in Theorem 3. Therefore, we have that

$$
b(G \otimes H) \geq \max \{b(G), b(H)\} .
$$

Now, for proving the upper bound of $b(G \square H)$, let $r=\operatorname{rad}(G), s=b(H)$, and $\left(x_{1}, \ldots, x_{s}\right)$ be an optimum burning sequence for $H$. Suppose that $u_{k}$ is a central node for $G$, and $H_{k}$ is the subgraph of $G \square H$ that is isomorphic to $H$ corresponding to $u_{k}$, as defined above. For $1 \leq i \leq s$, let $y_{i}=\left(u_{k}, x_{i}\right)$. We can easily see that $\left\{N_{r+s-i}\left[y_{i}\right]\right\}_{i=1}^{s}$ forms a covering for the node set of $G \square H$. Thus, by Theorem 2, we conclude that $b(G \square H) \leq s+r$. By symmetry, we have that $b(G \square H) \leq b(G)+\operatorname{rad}(H)$ and so the proof is finished.

Note that the lower bound in Theorem 7 is achieved by $K_{2} \square P_{n}$, where $n \in$ $\left\{k^{2}+1, k^{2}+2: k \in \mathbb{N}\right\}$. Also, it is achieved by $K_{2} \otimes P_{n}$, where $n$ is a square number. The upper bound is tight if $G$ is any graph of radius one and $H$ is a path of square order. For example, let $G=P_{3}$ and $H=P_{4}$. Then by Theorem 5 , we can show that $b(G \square H)>2$. On the other hand, by Theorem 7 , we have that $b(G \square H) \leq 3$. Therefore, we conclude that $b\left(P_{3} \square P_{4}\right)=3$, which is suggested by the upper bound in Theorem 7 .

The hypercube graph, or the $n$-cube, or the $n$-dimensional hypercube, denoted by $Q_{n}$, is the graph of order $2^{n}$ in which every node corresponds to a binary string of length $n$, and two nodes are adjacent if and only if their corresponding binary strings differ in exactly one bit. It is known (and easy to see) that $Q_{0}=K_{1}$, $Q_{1}=K_{2}$, and

$$
Q_{n}=Q_{n-1} \square K_{2} .
$$

Moreover, the diameter of $Q_{n}$ is $n$.

Suppose that we choose the nodes $x_{1}$ and $x_{2}$ in $Q_{n}$ with $d\left(x_{1}, x_{2}\right)=n$, and we take $k=\left\lceil\frac{n}{2}\right\rceil+1$. As $Q_{n}$ is a node transitive graph, without loss of generality, we may assume that $x_{1}$ is the node that corresponds to the binary string with all zero bits, and $x_{2}$ corresponds to the binary string with all one bits. We then can easily see that $V\left(Q_{n}\right)=N_{k-1}\left[x_{1}\right] \cup N_{k-2}\left[x_{2}\right]$. Thus, by Theorem 2 , we conclude that $b\left(Q_{n}\right) \leq k=\left\lceil\frac{n}{2}\right\rceil+1$. We have the following conjecture for the optimum burning of the hypercube graphs.

Conjecture 8. Let $n$ be a positive integer, and $k=\left\lceil\frac{n}{2}\right\rceil+1$. Then for the hypercube $Q_{n}$ we have that $b\left(Q_{n}\right)=k$. Moreover, if $n$ is even, then in any optimal burning sequence $\left(x_{1}, \ldots, x_{k}\right)$ for $Q_{n}$ we must have $d\left(x_{1}, x_{2}\right)=n=\operatorname{diam}\left(Q_{n}\right)$.

It is easy to check that the conjecture is true for $n \in\{1,2,3\}$ but it seems more challenging to prove it for any $n$. It is known that if $|n / 2-k|=o\left(n^{2 / 3}\right)$, then

$$
\left(\begin{array}{l}
n \\
k
\end{array}\right) \sim \frac{2^{n}}{\sqrt{\frac{1}{2} n \pi}} \mathrm{e}^{-\frac{(n-2 k)^{2}}{2 n}}
$$


see, for example, Section 5.4 in [5]. We use this to find a slightly weaker lower bound on the burning number of the hypercube graph $Q_{n}$. This lower bound leads to an asymptotic result for the burning number of the hypercube graph $Q_{n}$.

Theorem 9. For the hypercube graph $Q_{n}$, we have that

$$
b\left(Q_{n}\right) \sim n / 2 .
$$

Proof. We will prove this by showing that

$$
\frac{n}{2}+1-\sqrt{n \log n}<b\left(Q_{n}\right) \leq\left\lceil\frac{n}{2}\right\rceil+1
$$

As we mentioned earlier, by burning two nodes $x_{1}, x_{2} \in V\left(Q_{n}\right)$ with $d\left(x_{1}, x_{2}\right)=n$ in the first and second steps of a burning process for $Q_{n}$, we will have every node burning at time $t=\left\lceil\frac{n}{2}\right\rceil+1$. Therefore, $b\left(Q_{n}\right) \leq\left\lceil\frac{n}{2}\right\rceil+1$.

Now, assume that $k=\left\lceil\frac{n}{2}+1-c \sqrt{n \log n}\right\rceil$, where $c$ is a constant that will be determined later on in the proof. We want to show that burning $Q_{n}$ in $k$ steps is asymptotically impossible. Clearly, the number of nodes in the $r$-th closed neighbourhood of any node in $Q_{n}$ equals $\sum_{i=0}^{r}\left(\begin{array}{c}n \\ i\end{array}\right)$. Now, suppose that $\left(x_{1}, \ldots, x_{k}\right)$ is a sequence of nodes in $Q_{n}$. The total number of nodes that can be covered by $\bigcup_{i=1}^{k} N_{k-i}\left[x_{i}\right]$ is at most

$$
\sum_{i=1}^{k}\left|N_{k-i}\left[x_{i}\right]\right|=\sum_{i=1}^{k} \sum_{j=0}^{k-i}\left(\begin{array}{l}
n \\
j
\end{array}\right) \leq \sum_{i=1}^{k} \sum_{j=0}^{k-i}\left(\begin{array}{c}
n \\
k-1
\end{array}\right) \leq k^{2}\left(\begin{array}{c}
n \\
k-1
\end{array}\right),
$$

since $\left(\begin{array}{l}n \\ j\end{array}\right)$ is an increasing function of $j$ for $0 \leq j \leq k-1 \leq n / 2$.

By (2), we have that

$$
k^{2}\left(\begin{array}{c}
n \\
k-1
\end{array}\right) \sim \frac{n^{2}}{4} \cdot \frac{2^{n}}{\sqrt{\frac{1}{2} n \pi}} \mathrm{e}^{-\frac{(n-2 k+2)^{2}}{2 n}}=O\left(n^{3 / 2} 2^{n} \mathrm{e}^{-2 c^{2} \log n}\right),
$$

which is of order $o\left(2^{n}\right)$ if, for example, $c=1>\frac{\sqrt{3}}{2} \approx 0.866$. Thus, the lower bound in (3) holds and the proof is finished.

We now consider the burning number of a $m \times n$ strong grid, that is, the strong product of $P_{m}$ and $P_{n}$. Figure 2 shows an example of a $3 \times 3$ strong grid. Note that the $r$-th closed neighbourhood (in a strong grid $G$ ) of a node $x$ (that is at distance at least $r$ from the border of the grid) induces a smaller $(2 r+1) \times(2 r+1)$ strong grid centred at $x$. We call such an induced subgraph on precisely $(2 r+1)^{2}$ nodes a square of radius $r$. We have the following theorem about the burning number of strong grids. The technique used in proving this theorem is similar to the technique used for finding the burning number of the Cartesian grids presented in $[7]$. 


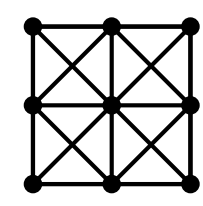

Figure 2. A $3 \times 3$ strong grid.

Theorem 10. Let $G$ be a $m \times n$ strong grid with $1 \leq m \leq n$, where $m=m(n)$ is a function of $n$. Then we have the following asymptotic results:

$$
b(G)= \begin{cases}\Theta\left(n^{1 / 2}\right) & \text { if } m=O\left(n^{1 / 2}\right) \\ (1+o(1))\left(\frac{3}{4}\right)^{1 / 3}(m n)^{1 / 3} & \text { if } m=\omega\left(n^{1 / 2}\right) .\end{cases}
$$

Proof. First, we show a lower bound by applying Lemma 1 as follows. Suppose that $\left(x_{1}, \ldots, x_{k}\right), k=b(G)$, is an optimal burning sequence for $G$. Thus, every node in $G$ must be in the $(k-i)$-th neighbourhood of a node $x_{i}$, for some $1 \leq i \leq k$. Therefore, it follows from (1) that

$$
\begin{aligned}
m n=|V(G)| & \leq\left|N_{k-1}\left[x_{1}\right]\right|+\left|N_{k-2}\left[x_{2}\right]\right|+\cdots+\left|N_{0}\left[x_{k}\right]\right| \\
& \leq \sum_{i=1}^{k}(2 i-1)^{2}=\frac{k(2 k-1)(2 k+1)}{3}=\frac{4 k^{3}}{3}-\frac{k}{3}<\frac{4 k^{3}}{3},
\end{aligned}
$$

and so

$$
b(G)=k>(3 / 4)^{1 / 3}(m n)^{1 / 3} .
$$

This bound is used when $m=\omega\left(n^{1 / 2}\right)$. On the other hand, if $m=O\left(n^{1 / 2}\right)$, then we use the fact that the path $P_{n}$ is an isometric subtree of $G$. It follows from Theorem 4 that

$$
b(G) \geq b\left(P_{n}\right) \geq n^{1 / 2},
$$

and hence the lower bounds are proved.

Now, let us move to the upper bounds. If $m<c_{1} \sqrt{n}$ for some constant $c_{1}$, then by Theorem 6 we may burn the path $P_{n}$ on the top border of $G$ in $\lceil\sqrt{n}\rceil$ steps. Since in this case every node in $G$ is within distance $c_{1} \sqrt{n}$ from some node on this path, after at most $c_{1} \sqrt{n}$ additional steps all nodes in $G$ must be burned. Therefore, we have that $b(G)=O(\sqrt{n})$ and the claimed upper bound in this case is proved.

It remains to concentrate on the case $m=\omega\left(n^{1 / 2}\right)$. Let $\alpha=\alpha(n)=m / n^{1 / 2}$; note that $1 \ll \alpha \leq n^{1 / 2}$ as $m \leq n$. In order to avoid boundary effects, it will be convenient to consider an infinite grid that a finite grid $G$ is part of. We are going to present a way to cover nodes of $G$ with a family of squares of successive 
radii that are placed on some vertical strips. The radii of the squares are going to range from $k_{1}$ to some $k_{2}$ which will turn out to be at most $k_{3}$, where

$$
\begin{aligned}
& k_{1}=\frac{(m n)^{1 / 3}}{\alpha^{1 / 6}}=n^{1 / 2} \alpha^{1 / 6} \text { and } \\
& k_{3}=\left(\frac{3}{4}\right)^{1 / 3}(m n)^{1 / 3}\left(1+\frac{1}{\alpha^{1 / 6}}\right)=\left(\frac{3}{4}\right)^{1 / 3} n^{1 / 2} \alpha^{1 / 3}\left(1+\frac{1}{\alpha^{1 / 6}}\right) .
\end{aligned}
$$

For simplicity, we do not round numbers that are supposed to be integers either up or down; this is justified since these rounding errors are negligible in the asymptomatic calculations we will make. Moreover, each radius in this range will appear exactly once in the proposed covering. By Theorem 2, we will get that $b(G) \leq k_{2}+1 \leq k_{3}+1 \sim\left(\frac{3}{4}\right)^{1 / 3}(m n)^{1 / 3}$, and the proof will be finished.

As already mentioned, in order to cover nodes of $G$ with a family of squares, it is natural to arrange the squares by putting them in vertical strips without making an effort to avoid overlaps between the squares. The first strip, $S_{1}$, of width $2 r_{1}+1=2 k_{1}+1$ will be covered (from top to bottom) with disjoint squares of radii $r_{1}, r_{1}+1, \ldots, r_{2}-1$ for some $r_{2}>r_{1}$. The squares will be put on the grid so that their right borders coincide with the border of the strip. On the other hand, as the radii are increasing, the squares will reach nodes away from the left border and so they will intersect with the squares that will be used to cover the next neighbouring strip. Such nodes will be called overlapping. Moreover, some part of the last square (of radius $r_{2}-1$ ) might fall outside of $G$ (but, of course, be part of an infinite grid). Nodes that are covered but are not part of $G$ will be called wasted. Once the first strip is covered, we move on to the next strip, $S_{2}$, of width $2 r_{2}+1$, and proceed as before using squares of radii between $r_{2}$ and $r_{3}-1$, etc. An example of a layout of this covering is presented in Figure 3 . We see a $m \times n$ strong grid $G$ with black nodes and edges in the plane (with white background). The strong grid $G$ is covered with blue squares of successive radii between $r_{1}$ (the radius of the first square on the top right corner of $G$ ) and $r_{\ell}$ (the radius of the last square on the left side of $G$ ). The centre node of each blue square is shown in red (representing a fire source). The grey rectangles represent the overlapping areas.

Our goal is to show that the total number of overlapping and wasted nodes is negligible comparing to the number of nodes of the finite grid $G$. Using the described strategy, we partition the strong grid $G$ (from right to left) into some vertical strips $S_{1}, S_{2}, \ldots, S_{\ell}$ (for some positive integer $\ell$ ), in which the radius of $S_{i}$ is $r_{i}$. The increasing sequence $k_{1}=r_{1}<r_{2}<\ldots<r_{\ell}<r_{\ell+1}$ is a function of $n$ and $m$ and is defined recursively. Note that the last square used in this covering has radius $k_{2}=r_{\ell+1}-1$, and so it follows from Theorem 2 that $b(G) \leq r_{\ell+1}$. It remains to show that $r_{\ell+1}-1 \leq k_{3}$. 


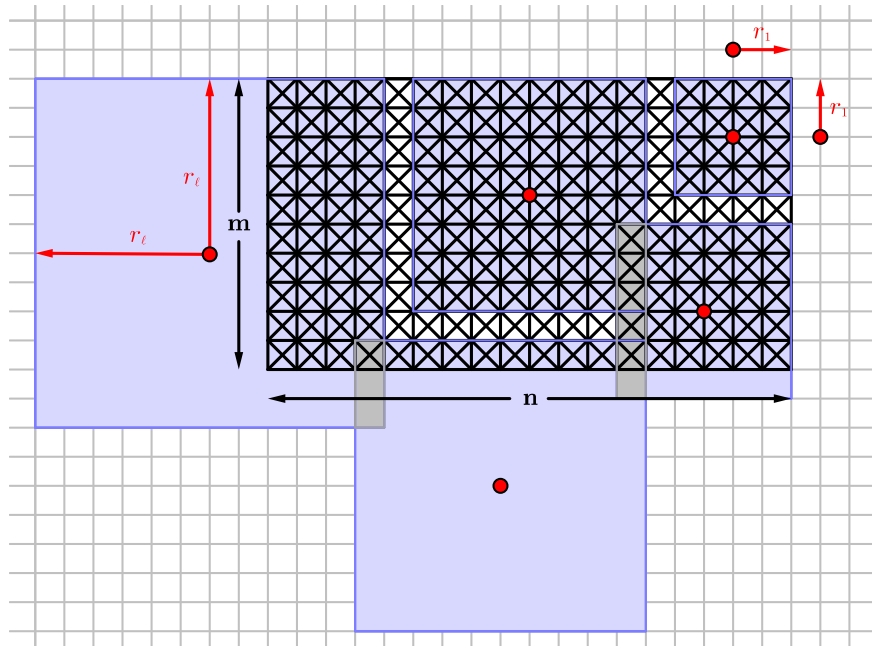

Figure 3. A covering of the $m \times n$ strong grid $G$.

It should be possible to estimate $\ell$ and $r_{i}$ 's but it seems rather complicated and quite tedious. Instead, for a contradiction, let us suppose that it is impossible to cover the grid with squares of radii between $k_{1}$ and $k_{3}$ (the way we described above); that is, we suppose that $k_{2}>k_{3}$. In other words, we suppose that the family of squares of radii $k_{1}, \ldots, k_{3}$ does not cover all nodes of $G$. Since $G$ has $m n$ nodes that are only partially covered by the family of squares of radii $k_{1}, \ldots, k_{3}$, we must have

$$
\sum_{i=k_{1}}^{k_{3}}(2 i+1)^{2}-E<m n
$$

where $E$ is the number of nodes that are either wasted or overlapping. We are going to estimate $E$ to get the desired contradiction.

Let us focus on any strip $S_{i}(1 \leq i \leq \ell-1)$ of width $2 r_{i}+1$ and length $m$ (the last strip, $S_{\ell}$, will be considered separately). Since the side length of each square is at least $2 r_{i}+1 \geq 2 k_{1}+1$, the total number of squares used to cover $S_{i}$ is at most

$$
\left\lceil\frac{m}{2 k_{1}+1}\right\rceil \leq \frac{m}{2 k_{1}}+1
$$

and hence we get $r_{i+1} \leq r_{i}+m /\left(2 k_{1}\right)+1$. The number of overlapping nodes in the union of squares used to cover $S_{i}$ that intersect with $S_{i+1}$ is at most $O_{i}$, where

$$
O_{i}=\left(\left(2\left(r_{i+1}-1\right)+1\right)-\left(2 r_{i}+1\right)\right) m \leq \frac{m^{2}}{k_{1}} .
$$

Since the radius of every square in the covering of $S_{i}$ is at most $r_{i+1}-1$, it is easy to see that the number of wasted nodes is at most $W_{i}$, where

$$
W_{i}=\left(2\left(r_{i+1}-1\right)+1\right)^{2} \leq\left(2 k_{3}+1\right)^{2} .
$$


It follows that the total number of overlapping or wasted nodes in the covering of the first $\ell-1$ strips is at most

$$
E_{1}=\sum_{i=1}^{\ell-1}\left(O_{i}+W_{i}\right) \leq\left(\frac{m^{2}}{k_{1}}+\left(2 k_{3}+1\right)^{2}\right) \ell .
$$

Moreover, since the width of every strip $S_{i}, 1 \leq i \leq \ell-1$, is at least $2 k_{1}+1$, we have

$$
\ell \leq \frac{n}{2 k_{1}+1} \leq \frac{n}{2 k_{1}}
$$

and so we conclude that

$$
E_{1} \leq\left(\frac{m^{2}}{k_{1}}+\left(2 k_{3}+1\right)^{2}\right) \frac{n}{2 k_{1}}
$$

Now, let us focus on the last strip, $S_{\ell}$. Since it is the last strip, there are no overlapping nodes. On the other hand, it might happen that almost all nodes associated with $S_{\ell}$ are wasted (we use $E_{2}$ to denote the number of them). We will use a trivial upper bound for $E_{2}$ :

$$
E_{2} \leq m\left(2 r_{\ell+1}+1\right) \leq m\left(2 k_{3}+1\right)
$$

Combining the obtained bounds we get that the total number of overlapping or wasted nodes can be estimated to be at most

$$
\begin{aligned}
E=E_{1}+E_{2} & \leq\left(\frac{m^{2}}{k_{1}}+\left(2 k_{3}+1\right)^{2}\right) \ell+m\left(2 r_{\ell+1}+1\right) \\
& \leq\left(\frac{m^{2}}{k_{1}}+\left(2 k_{3}+1\right)^{2}\right) \frac{n}{2 k_{1}}+m\left(2 k_{3}+1\right) \\
& =\left(n^{1 / 2} \alpha^{11 / 6}+O\left(n \alpha^{2 / 3}\right)\right) \frac{n^{1 / 2}}{2 \alpha^{1 / 6}}+O\left(m n^{1 / 2} \alpha^{1 / 3}\right) \\
& \leq\left(n \alpha^{5 / 6}+O\left(n \alpha^{2 / 3}\right)\right) \frac{n^{1 / 2}}{2 \alpha^{1 / 6}}+O\left(m n / \alpha^{2 / 3}\right) \sim \frac{m n}{2 \alpha^{1 / 3}} .
\end{aligned}
$$

Hence, the number of nodes of the grid covered is at least

$$
\begin{aligned}
\sum_{i=k_{1}}^{k_{3}}(2 i+1)^{2}-E & =\frac{4\left(k_{3}+1\right)^{3}}{3}-\frac{k_{3}+1}{3}-\frac{4 k_{1}^{3}}{3}+\frac{k_{1}}{3}-E \\
& >\frac{4 k_{3}^{3}}{3}-\frac{4 k_{1}^{3}}{3}-E \\
& =m n\left(1+\frac{1}{\alpha^{1 / 6}}\right)^{3}-O\left(\frac{m n}{\alpha^{1 / 2}}\right)-O\left(\frac{m n}{\alpha^{1 / 3}}\right) \\
& =m n+\left(1+o(1) \frac{3}{\alpha^{1 / 6}} m n,\right.
\end{aligned}
$$

which is larger than $m n$, the order of $G$. This contradicts our assumption that $k_{2}>k_{3}$, and the proof is finished. 
We now proceed with another lower bound on the burning number of the strong product of two connected graphs. By combining the following theorem and Theorem 10, we can find a lower bound on the burning number of the strong product of graphs in terms of their diameters.

Theorem 11. Let $G$ and $H$ be two connected graphs with diameters $d_{1}$ and $d_{2}$, respectively. Suppose that $P$ is a shortest path between two nodes of distance $d_{1}$ in $G$, and $Q$ is a shortest path between two nodes of distance $d_{2}$ in $H$. Then we have that

$$
b(G \otimes H) \geq b(P \otimes Q) .
$$

Proof. To prove this, we will show that the subgraph $P \otimes Q$ of $G \otimes H$ satisfies the condition in Theorem 3. We first need to prove that $P \otimes Q$ is an isometric subgraph of $G \otimes H$. Let $x, z$ be two nodes in $P$ and $y, w$ be two nodes in $Q$. Note that $d_{P}(x, z)=d_{G}(x, z)$ and $d_{Q}(y, w)=d_{H}(y, w)$. Thus, we have that

$$
\begin{aligned}
d_{P \bowtie Q}((x, y),(z, w)) & =\max \left\{d_{P}(x, z), d_{Q}(y, w)\right\} \\
& =\max \left\{d_{G}(x, z), d_{H}(y, w)\right\}=d_{G \bowtie H}((x, y),(z, w)) .
\end{aligned}
$$

Now, we will show that the second condition in Theorem 3 holds as follows. Let $(u, v)$ be a node in $V(G \otimes H) \backslash V(P \otimes Q)$, and $r$ be a positive integer. Also, assume that $A$ is the smallest connected subgraph of $P$ that contains $N_{G}^{r}[u] \cap P$. Clearly, $A$ must be a subpath of $P$ with end points in $N_{G}^{r}[u]$. Similarly, we define $B$ to be the smallest subpath of $Q$ that contains $N_{H}^{r}[v] \cap Q$.

Note that both $A$ and $B$ are of radius at most $r$ (as path graphs). To see this, assume that $x$ and $y$ are the end points of $A$ (as a path). Then we have that

$$
d_{A}(x, y)=d_{P}(x, y)=d_{G}(x, y) \leq d_{G}(x, u)+d_{G}(u, y) \leq r+r=2 r .
$$

Thus, $A$ is a path of length at most $2 r$, and consequently, the radius of $A$ must be at most $r$. Similarly, we can prove that $B$ is a path of radius at most $r$.

Now, we can easily see that $A \otimes B$ is a subgrid of $P \otimes Q$ with radius at most $r$, and its centre consists of either a single node or two adjacent nodes, or four nodes that are mutually adjacent. Namely, if the centres of $A$ and $B$ are $C_{1}$ and $C_{2}$, respectively, then the centre of $A \otimes B$ is $C_{1} \otimes C_{2}$. Moreover, note that $N_{G \otimes H}^{r}[(u, v)] \cap(P \otimes Q)$ is a subgraph of $A \otimes B$. To show this, assume that $(x, y)$ is a node in $N_{G \otimes H}^{r}[(u, v)] \cap(P \otimes Q)$. This implies that $x \in P, y \in Q$, and $d_{G \otimes H}((u, v),(x, y)) \leq r$. Thus, $\max \left\{d_{G}(u, v), d_{H}(v, y)\right\} \leq r$. This means that $d_{G}(u, v) \leq r$ and $d_{H}(v, y) \leq r$. Hence, we must have that $x \in A$ and $y \in B$. Therefore, $(x, y) \in A \otimes B$.

Thus, the above arguments imply that

$$
N_{G \otimes H}^{r}[(u, v)] \cap(P \otimes Q) \subseteq(A \otimes B)=N_{A \bowtie B}^{r}\left[\left(u_{0}, v_{0}\right)\right],
$$

where $\left(u_{0}, v_{0}\right)$ is a central node in $A \otimes B$. Hence, the conditions in Theorem 3 hold. Therefore, we conclude that

$$
b(G \otimes H) \geq b(P \otimes Q),
$$


and the proof is finished.

The following corollary follows immediately from Theorem 11 and the proof of Theorem 10 (see (4) and (5)).

Corollary 12. Let $G$ and $H$ be two connected graphs with diameters $d_{1}$ and $d_{2}$, respectively, such that $d_{1} \leq d_{2}$. Then we have that

$$
b(G \otimes H)>\max \left\{d_{2}^{1 / 2},(3 / 4)^{1 / 3}\left(d_{1} d_{2}\right)^{1 / 3}\right\} .
$$

We now move on to the burning number of $G \circ H$. Note that when $G$ is a single node, $G \circ H$ is isomorphic to $H$ and clearly, $b(G \circ H)=b(H)$. Hence, we consider $b(G \circ H)$ for $G$ being of order at least two. Here we give a simple characterization of $b(G \circ H)$ for $G$ being connected and of order at least two.

Theorem 13. Let $G$ be a connected graph of order at least two and $H$ be any graph. Then we have that

$$
b(G) \leq b(G \circ H) \leq b(G)+1 .
$$

Moreover, $b(G \circ H)=b(G)$ if and only if one of the following conditions holds.

(i) $b(H)=1$ or equivalently $H=K_{1}$.

(ii) $b(H)=2$ and $G$ has an optimum burning sequence $\left(x_{1}, \ldots, x_{k}\right)$ such that one of the neighbours of $x_{k}$ is burned in step $k-1$.

(iii) $G$ has an optimum burning sequence $\left(x_{1}, \ldots, x_{k}\right)$ such that each of $x_{k-1}$ and $x_{k}$ has a neighbour that is burned in step $k-1$.

Proof. Suppose that $V(G)=\left\{u_{1}, \ldots, u_{n}\right\}$, and $V(H)=\left\{v_{1}, \ldots, v_{m}\right\}$, where $m$ and $n$ are two positive integers. Note that the subgraph of $G \circ H$ induced by the nodes $\left\{\left(u_{1}, v_{1}\right),\left(u_{2}, v_{1}\right), \ldots,\left(u_{n}, v_{1}\right)\right\}$ is isomorphic to $G$. We call this subgraph $G_{1}$, and we claim that $G_{1}$ is an isometric subgraph of $G \circ H$ that satisfies the conditions in Theorem 3: to show this, suppose that $\left(u_{i}, v_{1}\right)$ and $\left(u_{j}, v_{1}\right)$ are two nodes in $G_{1}$, where $1 \leq i<j \leq n$. As we mentioned in Section 2,

$$
d_{G \circ H}\left(\left(u_{i}, v_{1}\right),\left(u_{j}, v_{1}\right)\right)=d_{G}\left(u_{i}, u_{j}\right)=d_{G_{1}}\left(u_{i}, u_{j}\right) .
$$

Hence, $G_{1}$ is an isometric subgraph of $G \circ H$. Now, suppose that $\left(u_{i}, v_{j}\right)$ is a node in $G \circ H$, with $j \neq 1$, and $r$ is a positive integer. We can easily see that $N_{r}^{G \circ H}\left[\left(u_{i}, v_{j}\right)\right] \cap V\left(G_{1}\right) \subseteq N_{r}^{G_{1}}\left[\left(u_{i}, v_{1}\right)\right]$. Thus, the claim is true. Therefore, by Theorem 3, we conclude that

$$
b(G \circ H) \geq b\left(G_{1}\right)=b(G),
$$

thus proving the first inequality.

Now, for proving the second inequality, assume that $b(G)=k$. Let $\left(x_{1}, \ldots, x_{k}\right)$ be an optimum burning sequence for $G$. For $1 \leq i \leq n$, assume that $H_{i}$ denotes the subgraph of $G \circ H$ that is isomorphic to $H$ and corresponds to the node $u_{i} \in G$. That is, $V\left(H_{i}\right)=\left\{\left(u_{i}, v\right): v \in V(H)\right\}$. Now, for $1 \leq j \leq k$, take $y_{j}=\left(x_{j}, v_{1}\right)$. Clearly, $\left(y_{1}, \ldots, y_{k}\right)$ forms a burning sequence for $G_{1}$. Thus, by the 
end of the $k$-th step, each $H_{i}$ contains the burning node $\left(u_{i}, v_{1}\right)$ (at least). Note that $\left\{V\left(H_{i}\right)\right\}_{i=1}^{n}$ forms a partition for the node set of $G \circ H$. Since by assumption, $G_{1}$ is connected and contains at least two nodes, by definition of the lexicographic product, we can see that after burning the sequence $\left(y_{1}, \ldots, y_{k}\right)$ every $H_{i}$ has a neighbour (that is, a node that is adjacent to all nodes in $H_{i}$ ) that is burning. Therefore, $\left\{N_{k+1-j}\left[y_{j}\right]\right\}_{j=1}^{k}$ forms a covering for the node set of $G \circ H$. Hence, by Theorem 2, we conclude that $b(G \circ H) \leq k+1=b(G)+1$.

We now claim that $b(G \circ H)=b(G)=k$ if and only if one of the conditions (i), (ii), and (iii) in theorem's statement holds. We first assume that one of the three conditions holds:

Case (i): If $H=K_{1}$, then $G \circ H=G$, and the statement of the theorem is clearly true.

Case (ii): Assume now that $b(H)=2$, and there is an optimum burning $\left(x_{1}, \ldots, x_{k}\right)$ for $G$ such that one of the neighbours of $x_{k}$ is burned in step $k-1$. Since $b(H)=2$, then $H$ is of order at least two and we have two possibilities: either $H$ is of radius one, or there are two non-adjacent nodes $x, y \in V(H)$ such that $V(H)=N[x] \cup\{y\}$. If the former holds, then without loss of generality, assume that $v_{1}$ is a central node in $H$. If the latter holds, then without loss of generality assume that $v_{1}$ and $v_{2}$ are two non-adjacent nodes in $H$ such that $V(H)=N\left[v_{1}\right] \cup\left\{v_{2}\right\}$. In the first case let $\left(y_{1}, \ldots, y_{k}\right)$ be the sequence in which $y_{j}=\left(x_{j}, v_{1}\right)$, for $1 \leq j \leq k$. In the second case, let $\left(y_{1}, \ldots, y_{k}\right)$ be the sequence in which $y_{j}=\left(x_{j}, v_{1}\right)$, for $1 \leq j \leq k-1$, and $y_{k}=\left(x_{k-1}, v_{2}\right)$. We claim that in both cases, $\left(y_{1}, \ldots, y_{k}\right)$ is a burning sequence for $G \circ H$. Now, from the assumptions, we can see that in both cases, every $H_{i}$ will have a neighbour that is burning after burning the nodes $\left(y_{1}, \ldots, y_{k-1}\right)$. Hence, every node in $G \circ H$ will be within distance one from a burning node by the end of step $k-1$. Therefore, $\left(y_{1}, \ldots, y_{k}\right)$ is a burning sequence for $G \circ H$.

Case (iii): This time we assume that $G$ has an optimum burning sequence $\left(x_{1}, \ldots, x_{k}\right)$ such that each of $x_{k-1}$ and $x_{k}$ has a neighbour that is burned in step $k-1$. If $b(H) \leq 2$, then by the above cases, we conclude that $b(G \circ H)=b(G)$. If $b(H) \geq 3$, then let $\left(y_{1}, \ldots, y_{k}\right)$ be the sequence in which $y_{j}=\left(x_{j}, v_{1}\right)$, for $1 \leq j \leq k$, and with $v_{1}$ being any node in $H$. Then similar to Case (ii) it is easy to see that $\left(y_{1}, \ldots, y_{k}\right)$ is a burning sequence for $G \circ H$.

We next aim at proving the converse direction. That is, we suppose $b(G \circ H)=$ $b(G)$, and our goal is to show that one of the three conditions must hold. On a high level, our goal is to provide an algorithm that modifies, if necessary, the burning sequence of $G \circ H$ in such a way that all fire sources (except for possibly one) belong to $G_{1}$ and each of the $H_{i}$ (except for possibly one) contains at most one fire source. After having performed these changes, we are able to show that one of the three conditions given in the statement of the theorem must be fulfilled. 
We explain the idea now in more detail. First note that since $G$ is of order at least two, by definition of the lexicographic product, for $2 \leq j \leq n$, every node in $H_{j}$ is adjacent to every node in $H_{1}$. Thus, the distance between every pair of distinct nodes $x, y \in V\left(H_{i}\right)$, where $1 \leq i \leq n$, is at most two in $G \circ H$. Moreover, by definition of the lexicographic product and the facts we mentioned in Section 2 , it is easy to see that $d(x, z)=d(y, z)$ for every node $z \in(G \circ H) \backslash H_{i}$. Thus, for every $r \geq 2$ and $s \geq 0$ with $s<r$,

$$
N_{s}[x] \subseteq N_{r}[x]=N_{r}[y] .
$$

Hence, by Lemma 1 , we conclude that for $1 \leq i \leq n$, there can be at most three fire sources in $H_{i}$. More precisely, there are five possibilities: either $y_{j}, y_{j+1}, y_{j+2} \in$ $V\left(H_{i}\right)$, or only $y_{j}, y_{j+1} \in V\left(H_{i}\right)$, or only $y_{j}, y_{j+2} \in V\left(H_{i}\right)$, or only $y_{j} \in V\left(H_{i}\right)$, or there is no fire source in $H_{i}$. As mentioned before, we will now modify the burning sequence (if necessary) to find a new burning sequence for $G \circ H$ in which all fire sources, except possibly $y_{k}$, are in $G_{1}$, and there is at most one fire source from each $H_{i}$, for $1 \leq i \leq n$, except possibly the one that contains $y_{k-1}$. This is made precise by the following algorithm (we denote the copy of $H$ in $G \circ H$ that contains $y_{i}$ by $H_{y_{i}}$, for $\left.1 \leq i \leq k\right)$ :

Algorithm 14. Suppose that $\left(y_{1}, \ldots, y_{k}\right)$ is a burning sequence for $G \circ H$. We then perform the following steps.

Stage 1. For $1 \leq j \leq k-1$, perform the following steps:

Stage 1.1. If $y_{j} \notin G_{1}$, that $i s, y_{j}=\left(u_{i}, v_{\ell}\right)$ for some $1 \leq i \leq n$ and $2 \leq l \leq m$, then do the following: if $y_{j+1}=\left(u_{i}, v_{1}\right)$, let $y_{j}=\left(u_{i}, v_{1}\right)$ and $y_{j+1}=\left(u_{i}, v_{\ell}\right)$ (switching $y_{j}$ and $\left.y_{j+1}\right)$. If $y_{j+2}=\left(u_{i}, v_{1}\right)$, then let $y_{j}=\left(u_{i}, v_{1}\right)$ and $y_{j+2}=\left(u_{i}, v_{\ell}\right)$ (switching $y_{j}$ and $\left.y_{j+2}\right)$. If $\left(u_{i}, v_{1}\right) \notin\left\{y_{j+1}, y_{j+2}\right\}$, then let $y_{j}=\left(u_{i}, v_{1}\right)$.

Stage 1.2. If $y_{j}, y_{j+1} \in V\left(H_{i}\right)$ for some $1 \leq i \leq n$ and $1 \leq j \leq k-2$, then select $x \in V\left(G_{1}\right) \backslash\left(\left(\cup_{i=1}^{j} N_{j-i}\left[y_{i}\right]\right) \cup\left(\cup_{i=1}^{j} H_{y_{i}}\right)\right)$ with $x \neq y_{\ell}$, for $\ell \geq j+2$.

Set $y_{j+1}=x$ in the sequence $\left(y_{1}, \ldots, y_{k}\right)$.

If $y_{k-1}, y_{k} \in V\left(H_{i}\right)$ for some $1 \leq i \leq n$ and $y_{k} \in\left(\cup_{i=1}^{k-1} N_{k-i}\left[y_{i}\right]\right)$, then select $x \in V\left(G_{1}\right) \backslash\left(\left(\cup_{i=1}^{k-1} N_{k-1-i}\left[y_{i}\right]\right) \cup\left(\cup_{i=1}^{k-1} H_{y_{i}}\right)\right)$.

Set $y_{k}=x$ in the sequence $\left(y_{1}, \ldots, y_{k}\right)$.

Stage 1.3. If $y_{j}, y_{j+2} \in V\left(H_{i}\right)$ for some $1 \leq i \leq n$, then select $x \in V\left(G_{1}\right) \backslash$ $\left(\left(\cup_{i=1}^{j+1} N_{j+1-i}\left[y_{i}\right]\right) \cup\left(\cup_{i=1}^{j+1} H_{y_{i}}\right)\right)$ with $x \neq y_{\ell}$, for $\ell \geq j+3$.

Set $y_{j+2}=x$ in the sequence $\left(y_{1}, \ldots, y_{k}\right)$.

Stage 2. If $y_{k-1} \in H_{i}$ and $y_{k}=\left(u_{j}, v_{\ell}\right)$ for some $1 \leq i, j \leq n$, with $i \neq j$, and $2 \leq \ell \leq m$, then set $y_{k}=\left(u_{j}, v_{1}\right)$ in the sequence $\left(y_{1}, \ldots, y_{k}\right)$.

Return the sequence $\left(y_{1}, \ldots, y_{k}\right)$.

We first show the following: 
Claim. Algorithm 14 returns a burning sequence for $G \circ H$ in which the first $k-1$ fire sources are all in $G_{1}$, and there is at most one fire source in each $H_{i}$, $1 \leq i \leq n$, except possibly one of them.

Proof of the claim. In Stage 1, we go through the first $k-1$ fire sources, and in every step we make sure that $y_{j}$ is in $G_{1}$. Each time that we find a second fire source chosen from the same $H_{i}$, where $1 \leq i \leq n$, we replace that fire source with a new one that is not in $H_{i}$, using the assumptions. As we show in the sequel, this happens for all $y_{j}$ 's except possibly $y_{k}$. In Stage 2 , we replace $y_{k}$ by a node from $G_{1}$ if possible. To prove this, we consider all possibilities as follows.

Part 1. For $1 \leq j \leq k-2$, suppose that $y_{j}=\left(u_{i}, v_{\ell}\right) \in H_{i}$, for some $1 \leq i \leq n$ and $2 \leq \ell \leq m$ (we consider $j=k-1$ in Part 2). Note that in this part $k-j \geq 2$. If $y_{j+1}$ (or $y_{j+2}$, respectively) is in $H_{i}$, then by (6), we conclude that $N_{k-j-1}\left[y_{j+1}\right] \subseteq$ $N_{k-j}\left[y_{j}\right]=N_{k-j}\left[\left(u_{i}, v_{1}\right)\right]\left(\right.$ or $N_{k-j-2}\left[y_{j+2}\right] \subseteq N_{k-j}\left[y_{j}\right]=N_{k-j}\left[\left(u_{i}, v_{1}\right)\right]$, respectively). Therefore, by switching the nodes suggested in Stage 1.1, and by Lemma 1 , the new sequence $\left(y_{1}, \ldots, y_{k}\right)$ is still a burning sequence for $G \circ H$.

Also, in Stage 1.2 we can find a node $x \in V\left(G_{1}\right) \backslash\left(\left(\cup_{i=1}^{j} N_{j-i}\left[y_{i}\right]\right) \cup\left(\cup_{i=1}^{j} H_{y_{i}}\right)\right)$ (similarly in Stage 1.3, $x \in V\left(G_{1}\right) \backslash\left(\left(\cup_{i=1}^{j+1} N_{j+1-i}\left[y_{i}\right]\right) \cup\left(\cup_{i=1}^{j+1} H_{y_{i}}\right)\right)$, respectively) with $x \neq y_{\ell}$, for $\ell \geq j+2(\ell \geq j+3$, respectively), since otherwise, the sequence $\left(y_{1}, \ldots, y_{j}\right)$ (or $\left(y_{1}, \ldots, y_{j+1}\right)$, respectively) must be a burning sequence for $G_{1}$ of length less than $k$, which is a contradiction. Thus replacing $y_{j+1}\left(y_{j+2}\right.$, respectively) by $x$ satisfies the conditions in Lemma 1. Hence, by the changes suggested to the sequence $\left(y_{1}, \ldots, y_{k}\right)$ in Stage 1.2 (Stage 1.3, respectively) of Algorithm 14, for $1 \leq j \leq k-2, y_{j}$ is the only fire source in $H_{y_{j}}$, and it is in $G_{1}$.

Part 2. For $j=k-1$, if $y_{k-1}, y_{k} \in H_{i}$, for some $1 \leq i \leq n$, then note that all nodes in $V\left(H_{i}\right)$ must be burned by the end of $k$-th step in the burning process: that is, only one step after burning $y_{k-1}$. Thus, either $N\left[y_{k-1}\right] \cup\left\{y_{k}\right\}=V\left(H_{i}\right)$ (equivalently, $b(H)=2$ ), or there must be a neighbour of $y_{k}$ in $(G \circ H) \backslash H_{i}$ (which is by definition of $G \circ H$ adjacent to all nodes in $V\left(H_{i}\right)$ ) that is burned in step $k-1$.

Case 2.1. If $N\left[y_{k-1}\right] \cup\left\{y_{k}\right\}=V\left(H_{i}\right)$ (that is, $b(H)=2$ ) and no neighbour of $y_{k}$ in $(G \circ H) \backslash H_{i}$ is burned in step $k-1$, then either $y_{k} \in N\left[y_{k-1}\right]$ (in case $H$ is of radius one), or $d\left(y_{k}, y_{k-1}\right)=2$. In both cases we must have already $y_{k-1}=\left(u_{i}, v_{1}\right)$, as otherwise, $V\left(H_{i}\right)$ cannot be burned by the end of $k$-th step. Thus, nothing happens in Stage 1.1 of these two cases. We now consider the two mentioned possibilities as follows.

Case 2.1.1. If $y_{k} \in N\left[y_{k-1}\right]$ ( $H$ is of radius one), then there must be a node $x \in V\left(G_{1}\right) \backslash\left(\left(\cup_{i=1}^{k-1} N_{k-1-i}\left[y_{i}\right]\right) \cup\left(\cup_{i=1}^{k-1} H_{y_{i}}\right)\right)$, since otherwise, the subsequence $\left(y_{1}, \ldots, y_{k-1}\right)$ must be a burning sequence for $G_{1}$, which is a contradiction. Hence, by Lemma 1, in Stage 1.2, we are allowed to replace $y_{k}$ by $x$. Thus, in this case $y_{k} \in G_{1}$. Therefore, all fire sources in the sequence $\left(y_{1}, \ldots, y_{k}\right)$ (that is returned 
as the output of Algorithm 14) are in $G_{1}$ and each $H_{j}, 1 \leq j \leq n$, contains at most one fire source.

Case 2.1.2. If $d\left(y_{k}, y_{k-1}\right)=2$, then $y_{k} \notin\left(\cup_{i=1}^{k-1} N_{k-i}\left[y_{i}\right]\right)$. Therefore, to maintain the conditions in Lemma 1, we are not allowed to replace $y_{k}$ by any other node in Stage 1.2 and Stage 2. Thus, in such a case Algorithm 14 returns a burning sequence in which all the first $k-1$ fire sources are in $G_{1}$, and each $H_{j}$, $1 \leq j \leq n$, except $H_{i}$ that contains $y_{k-1}$ and $y_{k}$, contains at most one fire source.

Case 2.2. If there is a node in $(G \circ H) \backslash H_{i}$ that is adjacent to all nodes in $V\left(H_{i}\right)$ and is burned in step $k-1$, then it means $y_{k-1}, y_{k} \in\left(\cup_{j=1}^{k-2} N_{k-j}\left[y_{j}\right]\right)$. Thus, by Lemma $1, y_{k-1}$ and $y_{k}$ can be any two nodes in $V\left(H_{i}\right)$, and therefore, the switching suggested in Stage 1.1 gives a new burning sequence for $G \circ H$. Also, in this case, there must be a node $x \in V\left(G_{1}\right) \backslash\left(\left(\cup_{j=1}^{k-1} N_{k-1-j}\left[y_{j}\right]\right) \cup\left(\cup_{j=1}^{k-1} H_{y_{j}}\right)\right)$, since otherwise, the subsequence $\left(y_{1}, \ldots, y_{k-1}\right)$ must be a burning sequence for $G_{1}$, which is a contradiction. Hence, by Lemma 1, in Stage 1.2 we are allowed to replace $y_{k}$ by $x \in G_{1}$. Thus in this case, Algorithm 14 returns a burning sequence $\left(y_{1}, \ldots, y_{k}\right)$ in which all fire sources are in $G_{1}$ and each $H_{j}, 1 \leq j \leq n$, contains at most one fire source.

Finally, in Stage 2, we check if we can choose $y_{k} \in G_{1}$ in case $y_{k}$ and $y_{k-1}$ are not in the same $H_{i}$. Therefore, after performing Algorithm 14 we have a burning sequence $\left(y_{1}, \ldots, y_{k}\right)$ for $G \circ H$ in which all fire sources, except possibly $y_{k}$, are in $G_{1}$, and there is at most one fire source in $H_{i}$, for $1 \leq i \leq n$, except possibly the one that contains $y_{k-1}$ and $y_{k}$. This finishes the proof of the claim.

We now come back to the proof of the converse direction. If $H$ is of order one, the result is trivial, so we may assume that $b(H) \geq 2$. There are now two possibilities: either $b(H)=2$ or $b(H) \geq 3$.

Case 1. If $b(H)=2$, then either $H$ is of radius one, or $V(H)=N\left[v_{1}\right] \cup\left\{v_{2}\right\}$ for non-adjacent nodes $v_{1}$ and $v_{2}$.

Case 1.1. If $H$ is of radius one, then assume that $v_{1}$ is a central node in $H$. By Case 2.1.1 of the proof of the claim, we know that all fire sources in the sequence $\left(y_{1}, \ldots, y_{k}\right)$ are in $G_{1}$ (and in distinct $H_{j}$ 's). Thus, by Lemma 1 , we have that

$$
V\left(G_{1}\right) \subseteq V(G \circ H)=\cup_{j=1}^{k} N_{k-j}\left[y_{j}\right]=\left(\cup_{j=1}^{k-1} N_{k-j}\left[y_{j}\right]\right) \cup\left\{y_{k}\right\} .
$$

It implies that $\left(y_{1}, \ldots, y_{k}\right)$ is also a burning sequence for $G_{1}$. Let $y_{k}=\left(u_{i}, v_{1}\right) \in$ $H_{i}$, for some $1 \leq i \leq n$. We know that $V\left(H_{i}\right) \backslash\left\{y_{k}\right\}$ contains a node $y=\left(u_{i}, v_{\ell}\right)$ (since $b(H)=2$ ). Note that $y_{k}$ is the only fire source in $H_{i}$. Thus, $y$ must have been burned only in step $k$ : indeed, otherwise, $y$ would have received the fire in an earlier step from a burning neighbour $x$ in $(G \circ H) \backslash H_{i}$. But then, by definition of the lexicographic product, $x$ must be also a neighbour of $y_{k}$, which is a contradiction ( since $y_{k}$ also must have received the fire from that node before step $k$ ). Now, in order to have $y$ burned in step $k$, there must be a neighbour of $y$ in $(G \circ H) \backslash H_{i}$ that is burned in step $k-1$. Let $x$ be such a neighbour, and 
assume that $x=\left(u_{j}, v_{r}\right) \in H_{j}$, where $1 \leq j \leq n$ with $j \neq i$, and $1 \leq r \leq m$. If $x$ is a fire source, then obviously $x \in G_{1}$. If $x$ is not a fire source, then for some $1 \leq s \leq k-2, x \in N_{k-s}\left[y_{s}\right]$, since it is burned in step $k-1$. By definition of the lexicographic product, this implies that every node in $H_{j}$ including $\left(u_{j}, v_{1}\right)$ must be also burned in step $k-1$. Note that $\left(u_{j}, v_{1}\right) \in G_{1}$ is also a neighbour of $y_{k}$ (by definition of the lexicographic product). Hence, $\left(y_{1}, \ldots, y_{k}\right)$ is a burning sequence for $G_{1}$ in which $y_{k}$ has a neighbour (in $G_{1}$ ) that is burned in step $k-1$, and condition (ii) is satisfied in this case.

Case 1.2. If $V(H)=N\left[v_{1}\right] \cup\left\{v_{2}\right\}$ for non-adjacent nodes $v_{1}$ and $v_{2}$, then assume that $y_{k-1} \in H_{i}$, where $1 \leq i \leq n$. There are two possibilities: either $y_{k}$ is in $H_{i}$, or not.

Case 1.2.1. If $y_{k} \in H_{i}$, then note that by Case 2.1 .2 of the proof of the claim, the first $k-1$ fire sources are in $G_{1}$ and each $H_{j}, 1 \leq j \leq n$, except $H_{i}$, contains at most one fire source. By Lemma 1,

$$
V\left(G_{1}\right) \subseteq V(G \circ H)=\cup_{j=1}^{k} N_{k-j}\left[y_{j}\right]=\left(\cup_{j=1}^{k-1} N_{k-j}\left[y_{j}\right]\right) \cup\left\{y_{k}\right\} .
$$

Now, note that there must be a node $x \in V\left(G_{1}\right) \backslash\left(\left(\cup_{j=1}^{k-1} N_{k-1-j}\left[y_{j}\right]\right) \cup\left(\cup_{j=1}^{k-1} H_{y_{j}}\right)\right)$, as otherwise, the sequence $\left(y_{1}, \ldots, y_{k-1}\right)$ is a burning sequence for $G_{1}$, which is a contradiction. Let $x \in H_{\ell}$, where $1 \leq \ell \leq n$, and $\ell \neq i$. Since $x$ must be burned in $k$-th step, there must be a neighbour of $x$ in $\cup_{j=1}^{k-1} N_{k-1-j}\left[y_{j}\right]$ (the set of nodes that are burning by the end of step $k-1$ ) that is burned in step $k-1$. Thus,

$$
V\left(G_{1}\right) \subseteq\left(\cup_{j=1}^{k-1} N_{k-j}\left[y_{j}\right]\right) \cup\{x\} .
$$

Hence, by Lemma 1 , we conclude that the sequence $\left(y_{1}, \ldots, y_{k-1}, x\right)$ forms a burning sequence for $G_{1}$. Moreover, a neighbour of $x$ is burned in step $k-1$, and condition (ii) is satisfied in this case as well.

Case 1.2.2. If $y_{k} \notin H_{i}$, then observe that in this case $\left(y_{1}, \ldots, y_{k}\right)$ is a burning sequence for $G \circ H$ such that all $y_{j}$ 's are in $G_{1}$. Thus, with an analogous argument to the case where $H$ is of radius one we conclude that $\left(y_{1}, \ldots, y_{k}\right)$ is also a burning sequence for $G_{1}$ in which $y_{k}$ has a neighbour (in $G_{1}$ ) that is burned in step $k-1$, and condition (ii) is also satisfied in this case.

Case 2. If $b(H) \geq 3$, then let $y_{k-1} \in H_{i}$, for some $1 \leq i \leq n$. We have then two possibilities: either $y_{k} \in H_{i}$, or not. However, by Case 2.2 of the proof of the claim, we can see that in such a case having $y_{k} \in H_{i}$ in the sequence returned by Algorithm 14 is impossible. That is, all fire sources in the sequence $\left(y_{1}, \ldots, y_{k}\right)$ are in $G_{1}$, and there is at most one fire source in each $H_{j}, 1 \leq j \leq n$. Moreover, there must be a neighbour of $y_{k-1}$ in $(G \circ H) \backslash H_{i}$ that is burned in step $k-1$. Let $x$ be such a neighbour, and assume that $x=\left(u_{j}, v_{r}\right) \in H_{j}$, where $1 \leq j \leq n$ with $j \neq i$, and $1 \leq r \leq m$. If $x$ is a fire source, then obviously $x \in G_{1}$. If $x$ is not a fire source, then for some $1 \leq s \leq k-2, x \in N_{k-s}\left[y_{s}\right]$, since it is burned in step $k-1$. By definition of the lexicographic product, this implies that node 
$\left(u_{j}, v_{1}\right)$ in $H_{j} \cap G_{1}$ must be also burned in step $k-1$. Note that $\left(u_{j}, v_{1}\right)$ is also a neighbour of $y_{k-1}$. Hence $y_{k-1}$ has a neighbour in $G_{1}$ that is burned in step $k-1$. Further, by Lemma $1, V\left(G_{1}\right) \subseteq V(G \circ H)=\cup_{j=1}^{k} N_{k-j}\left[y_{j}\right]$. Thus, the sequence $\left(y_{1}, \ldots, y_{k}\right)$ is a burning sequence for $G_{1}$ as well. Now, assume that $y_{k} \in H_{\ell}$, where $1 \leq \ell \leq n$ (with $i \neq \ell$ ). Again note that $H_{\ell}$ is of order at least two, and therefore, $V\left(H_{\ell}\right) \backslash\left\{y_{k}\right\}$ contains a node $y$. Using an analogous argument to the one in the case where $H$ is of radius one, we conclude that $y_{k}$ must have a neighbour in $G_{1}$ that is burned in step $k-1$. Thus $\left(y_{1}, \ldots, y_{k}\right)$ is a burning sequence for $G_{1}$ in which each of $y_{k-1}$ and $y_{k}$ has a neighbour that is burned in step $k-1$. Hence, condition (iii) is satisfied in this case, and the proof is finished.

We finish with an example showing how to apply Thorem 13. By Theorem 6, we know that $b\left(P_{n}\right)=\lceil\sqrt{n}=k$. By the proof of Theorem 6 given in [8], we can easily see that $P_{n}$ has an optimum burning sequence $\left(x_{1}, \ldots, x_{k}\right)$ such that one of the neighbours of $x_{k}$ is burned before the $k$-th step if and only if $n<k^{2}$. Moreover, if $n \leq k^{2}-2$, then $P_{n}$ has an optimum burning sequence $\left(x_{1}, \ldots, x_{k}\right)$ such that each of $x_{k}$ and $x_{k-1}$ has a neighbour that is burned in step $k-1$. Thus, by Theorem 13, $b\left(P_{n} \circ H\right)=b\left(P_{n}\right)=\lceil\sqrt{n}\rceil$ if and only if one of the following conditions holds:

(i) $H=K_{1}$.

(ii) $b(H)=2$ and $n=k^{2}-1$.

(ii) $n \leq k^{2}-2$ and $H$ is any graph.

If $n=k^{2}$, then in every optimum burning sequence $\left(x_{1}, \ldots, x_{k}\right)$ of $P_{n}$ all neighbours of $x_{k-1}$ and $x_{k}$ are burned in the $k$-th step. Therefore, by Theorem 13, in such a case $b\left(P_{n} \circ H\right)=b\left(P_{n}\right)+1=k+1$, with $H$ being any graph of order at least two.

\section{REFERENCES}

[1] S. Bessy, A. Bonato, J. Janssen, D. Rautenbach, E. Roshanbin, Bounds on the Burning Number, Discrete Applied Mathematics 235 (2018) 16-22.

[2] S. Bessy, A. Bonato, J. Janssen, D. Rautenbach, E. Roshanbin, Burning a Graph is Hard, Discrete Applied Mathematics 232 (2017) 73-87.

[3] A. Bonato, J. Janssen, E. Roshanbin, Burning a Graph as a Model of Social Contagion, Lecture Notes in Computer Science 8882, (2014), 13-22.

[4] A. Bonato, J. Janssen, E. Roshanbin, How to burn a graph, Internet Mathematics 1-2 (2016) 85-100.

[5] L. Florescu, J. Spencer, Asymptopia, Student Mathematical Library 71, American Mathematical Society, Providence, RI, 2014.

[6] W. Imrich, S. Klavžar, Product Graphs-Structure and Recognition, Wiley-Interscience Series in Discrete Mathematics and Optimization, Wiley-Interscience, New York, 2000.

[7] D. Mitsche, P. Prałat, E. Roshanbin, Burning graphs - a probabilistic perspective, Graphs and Combinatorics 33(2) (2017), 449-471.

[8] E. Roshanbin, Burning a graph as a model of social contagion, PhD Thesis, Dalhousie University, 2016. 
[9] D.B. West, Introduction to Graph Theory, 2nd edition, Prentice Hall, 2001.

Université de Nice Sophia-Antipolis, Laboratoire J-A Dieudonné, Parc ValROSE, 06108 NICE CEDEX 02

E-mail address: dmitsche@unice.fr

Department of Mathematics, Ryerson University, Toronto, ON, Canada

E-mail address: pralat@ryerson.ca

Department of Mathematics and Statistics, Dalhousie University, Halifax, NS, Canada, B3H 3J5

E-mail address: e.roshanbin@dal.Ca 